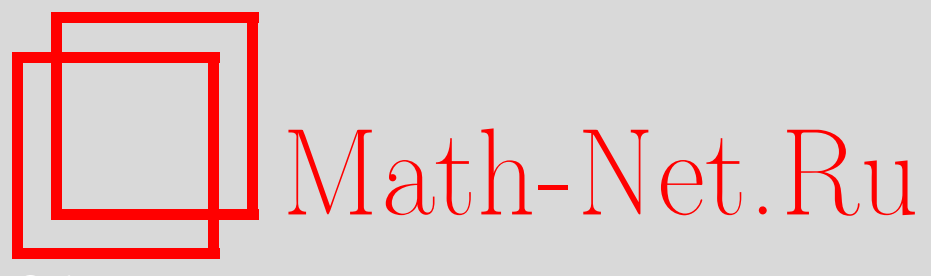

Л. А. Калякин, Устойчивость недиссипативных систем относительно постоянно действующих случайных возмущений, Матем. заметки, 2012, том 92, выпуск 1, 145148

DOI: https://doi.org/10.4213/mzm9487

Использование Общероссийского математического портала Math-Net.Ru подразумевает, что вы прочитали и согласны с пользовательским соглашением http://www . mathnet.ru/rus/agreement

Параметры загрузки:

IP : 54.92 .164 .108

26 апреля 2023 г., 10:12:21

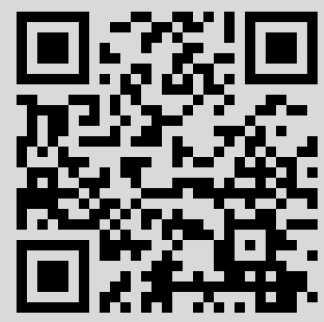




\section{Устойчивость недиссипативных систем относительно постоянно действующих случайных возмущений}

\section{Л. А. Калякин}

Эта работа возникла из попытки выяснить устойчивость явления авторезонанса [1] при малых случайных возмущениях. Оказалось, что известные результаты об устойчивости [2] не применимы из-за наличия в невозмущенной системе решений разного типа - ограниченных и неограниченных на бесконечности. Простейший пример такого рода дает задача о возмущении уравнения $\dot{x}=x(x-1)$, решения которого помимо устойчивого равновесия $x=0$ содержат траектории, уходящие на бесконечность. Вопрос состоит в том, при каких случайных возмущениях устойчивость сохраняется.

В общей постановке рассматривается система дифференциальных уравнений

$$
\frac{d \mathbf{x}}{d t}=\mathbf{f}(\mathbf{x}, t), \quad \mathbf{x} \in \mathbb{R}^{n}, \quad t>t_{0}
$$

с условием $\mathbf{f}(0, t) \equiv 0$. Возмущенная система записывается в виде

$$
\frac{d \mathbf{x}}{d t}=\mathbf{f}(\mathbf{x}, t)+\mu \mathbf{g}(\mathbf{x}, t, \omega), \quad t>t_{0}, \quad|\mu| \ll 1, \quad \omega \in \Omega .
$$

Здесь $\mathbf{g}(\mathbf{x}, t, \omega)$ - случайный процесс со значениями в $\mathbb{R}^{n}$, определенный на вероятностном пространстве $(\Omega, \mathscr{U}, \mathrm{P})$. Случай "белого шума" не рассматривается, так что уравнение понимается в обычном смысле [2; с. 26]. Класс возмущений ограничен требованием существования глобального решения с начальными данными из окрестности равновесия. Достаточные условия для выполнения этого требования касаются структуры нелинейности по $\mathbf{x}$; они хорошо известны $[2 ;$ с. 20$]$ и здесь не обсуждаются. Выделение малого параметра $\mu \in \mathbb{R},|\mu| \ll 1$ оказывается удобным приемом при анализе задачи с постоянно действующим возмущением, см. [3].

ОПРЕДЕЛЕНИЕ. Решение $\mathbf{x}(t) \equiv 0$ для системы (1) устойчиво по вероятности относительно постоянно действующих случайных возмущений равномерно на множестве $\mathscr{G}$, если $\forall \varepsilon, \nu>0 \exists \delta, \Delta: \forall\left|\mathbf{x}_{0}\right|<\delta,|\mu|<\Delta$ и $\forall \mathbf{g}(\mathbf{x}, t, \omega) \in \mathscr{G}$ решение $\mathbf{x}=\mathbf{x}_{g}(t, \omega)$ системы (2) с начальным условием $\left.\mathbf{x}\right|_{t=t_{0}}=\mathbf{x}_{0}$ обладает свойством $\mathrm{P}\left(\sup _{t>t_{0}}\left|\mathbf{x}_{g}(t, \omega)\right|>\varepsilon\right)<\nu$.

Обычно задача об устойчивости решается при условии равномерной по $t$ малости математического ожидания $\mathrm{M}\left[\mu \sup _{\mathbf{x}}|\mathbf{g}(\mathbf{x}, t, \omega)|\right] ;$ см. [2; c. 50]. При этом основным ограничением на исходные данные является требование диссипативности невозмущенной системы, которое можно отождествить с существованием глобальной функции Ляпунова $U(\mathbf{x}, t)$, т.е. во всем пространстве $\mathbf{x} \in \mathbb{R}^{n}[2 ;$ с. 24,25$]$. С одной стороны, такое условие оказывается весьма жестким и не выполняется, например, при наличии в невозмущенной системе траекторий, уходящих на бесконечность. С другой стороны, легко строятся примеры (см. [2; с.47], в которых отсутствие диссипативности ведет к неустойчивости с вероятностью единица. Отойти от условия диссипативности можно, если изменить класс возмущений, накладывая интегральные по $t$ ограничения для математического ожидания.

Ограничения на исходные данные. Предполагается, что в окрестности равновесия существует функция Ляпунова $U(\mathbf{x}, t)$, у которой производная в силу системы (1) определенно-отрицательная:

$$
m_{-}|\mathbf{x}|^{2}<U(\mathbf{x}, t)<m_{+}|\mathbf{x}|^{2}, \quad\left|\frac{\partial U}{\partial \mathbf{x}}\right|<m_{1}|\mathbf{x}|,\left.\quad \frac{d U}{d t}\right|_{(1)}<-\gamma U(\mathbf{x}, t) .
$$

Работа выполнена при поддержке Российского фонда фундаментальных исследований (гранты №№ 10-01-00186, 11-02-97003). 
Здесь все неравенства выполняются равномерно в области $|\mathbf{x}|<r, t>t_{0}$ при некоторых положительных константах $0<m_{ \pm}, m_{1}, \gamma, r<\infty$.

Рассматриваемый нами класс возмущений (случайных функций) выделяется наличием у каждой из них мажоранты: $|\mathbf{g}(\mathbf{x}, t, \omega)| \leqslant \eta(t, \omega) \forall|\mathbf{x}| \leqslant r, t \geqslant t_{0}$, которая стабилизируется на бесконечности к $\eta(\omega) \geqslant 0$, так что

$$
\text { для } I_{\eta}(\omega)=\int_{t_{0}}^{\infty}|\eta(\zeta, \omega)-\eta(\omega)| d \zeta \quad \text { выполняется } \quad \mathrm{P}\left(I_{\eta}(\omega)<\infty\right)=1 .
$$

В последнем условии содержится основное отличие от известных работ об устойчивости в системах со случайными возмущениями. Детерминированные возмущения подобного типа рассматривались в рамках так называемой интегральной устойчивости [4], [5]. Более широкий класс детерминированных возмущений с интегральным условием ограниченности среднего по $t$ исследован в [6], [3]. Требование ограниченности в среднем по $t$ было приспособлено для случайных возмущений при анализе устойчивости диссипативной системе [2; c. 52]. Однако, для недиссипативной системы при такого типа случайных возмущениях устойчивость не гарантируется. В данной работе условия накладываются не на среднее, а на интеграл $I_{\eta}(\omega)$.

Ограничения, связанные со случайностью возмущений, накладываются на мажоранту и описываются в терминах математического ожидания:

$$
\mathrm{M}[\eta(\omega)]<\infty, \quad \mathrm{M}\left[\exp \left(I_{\eta}(\omega)\right)\right]<\infty .
$$

Теорема. Если выполнены условия (3)-(5), то при $\forall R_{1}, R_{2} \in(0, \infty)$ равновесие $\mathbf{x}(t) \equiv 0$ системы (1) устойчиво по вероятности равномерно относительно случайных возмущений $\mathbf{g}(\mathbf{x}, t, \omega)$, для которых существует глобальное решение задачи Коши и $\mathrm{M}[\eta(\omega)] \leqslant R_{1}$, $\mathrm{M}\left[\exp \left(I_{\eta}(\omega)\right)\right] \leqslant R_{2}$.

Первая часть доказательства похожа на рассуждения, приведенные в [6]. Пусть выполнено $R_{1}, R_{2}>0$, а также $\varepsilon, \nu>0$ - произвольные положительные числа. Для системы (2) строится аналог функции Ляпунова в виде $V_{\eta}(\mathbf{x}, t, \omega)=U(\mathbf{x}, t) \exp \left(\beta_{\eta}(t, \omega)\right),|\mathbf{x}| \leqslant r, t>t_{0}$. Показатель определяется по формуле

$$
\beta_{\eta}(t, \omega)=-\frac{\gamma \nu}{R_{1}} \int_{t_{0}}^{t}[\eta(\zeta, \omega)-\eta(\omega)] d \zeta
$$

При доказательстве теоремы можно считать (не ограничивая общность ввиду данного выше определения): $\gamma \nu / R_{1}<1$, так что $\left|\beta_{\eta}(t, \omega)\right| \leqslant I_{\eta}(\omega) \forall t>t_{0}$.

Такой выбор $\beta_{\eta}(t, \omega)$, с одной стороны, обеспечивает в силу (3) две оценки:

$$
m_{-} \exp \left(-I_{\eta}(\omega)\right)|\mathbf{x}|^{2}<V_{\eta}(\mathbf{x}, t, \omega)<m_{+} \exp \left(I_{\eta}(\omega)\right)|\mathbf{x}|^{2}, \quad \forall|\mathbf{x}|<r, \quad t>t_{0} .
$$

Отсюда при любом $\varepsilon>0$ получается неравенство

$$
\sup _{|\mathbf{x}| \leqslant \delta_{\eta}, t>t_{0}} V_{\eta}(\mathbf{x}, t, \omega)<\inf _{|\mathbf{x}|=\varepsilon, t>t_{0}} V_{\eta}(\mathbf{x}, t, \omega) \stackrel{\text { def }}{=} V_{\eta, \varepsilon}(\omega),
$$

если выбрать границу шара: $\delta_{\eta}=\delta_{\eta}(\varepsilon, \omega) \stackrel{\text { def }}{=} \varepsilon \exp \left(-I_{\eta}(\omega)\right) \sqrt{m_{-} / m_{+}}$.

С другой стороны, производная, вычисленная в силу системы (2):

$$
\left.\frac{d V_{\eta}}{d t}\right|_{(2)}=\left[\left.\frac{d U}{d t}\right|_{(1)}+\mu \mathbf{g} \partial_{\mathbf{x}} U\right] \exp \left(\beta_{\eta}\right)+V_{\eta} \partial_{t} \beta_{\eta}(t, \omega),
$$


будет неположительна в кольцевой области $\delta_{g}<|\mathbf{x}|<\varepsilon$, если параметр возмущения $\mu$ достаточно мал. В самом деле, в силу условий теоремы при $|\mu|<\Delta_{g}$ для возмущений с предельной мажорантой $\eta(\omega) \leqslant R_{1} / \nu$ получаем

$$
\left.\frac{d V_{\eta}}{d t}\right|_{(2)} \leqslant\left[-\gamma+\frac{\Delta_{\eta}}{\delta_{\eta}} \frac{R_{1}}{\nu} \frac{m_{1}}{m_{-}}\right] V_{\eta}+\left[\frac{\Delta_{\eta}}{\delta_{\eta}} \frac{m_{1}}{m_{-}}\left(\eta_{g}(t, \omega)-\eta(\omega)\right)+\partial_{t} \beta_{\eta}(t, \omega)\right] V_{\eta} .
$$

Первое слагаемое здесь обращается в нуль, если положить

$$
\Delta_{\eta}(\varepsilon, \omega) \stackrel{\text { def }}{=} \delta_{\eta}(\varepsilon, \omega) \frac{\gamma \nu m_{-}}{R_{1} m_{1}}=\varepsilon \frac{\gamma \nu \exp \left(-I_{\eta}(\omega)\right)}{R_{1}} \frac{m_{-}}{m_{1}} \sqrt{\frac{m_{-}}{m_{+}}} ;
$$

после этого второе слагаемое равно нулю в силу выбора функции $\beta_{\eta}(t, \omega)$.

При таком выборе параметров $\delta_{\eta}, \Delta_{g}$ и при возмущении $\mathbf{g}(\mathbf{x}, t, \omega)$ из класса функций с ограниченными величинами $I_{\eta}(\omega), \eta(\omega)$ функция $V_{\eta}(\mathbf{x}, t, \omega)$ обладает свойством (6), а ее производная $d V_{\eta} /\left.d t\right|_{(2)}$ неположительна в кольце $\delta_{\eta}<|\mathbf{x}|<\varepsilon$ при $|\mu|<\Delta_{\eta}$. Отсюда следует, что функция $V_{\eta}(\mathbf{x}, t, \omega)$ на траектории, стартующей из внутреннего шара $\left|\mathbf{x}_{0}\right|<\delta_{\eta}$, не достигает минимального значения $V_{\eta, \varepsilon}(\omega)$ на внешней границе $|\mathbf{x}|=\varepsilon$. Следовательно, траектория $\mathbf{x}=\mathbf{x}_{g}(t, \omega)$ системы (2) остается внутри внешней сферы: $|\mathbf{x}|<\varepsilon$ при всех временах $t>t_{0}$. Для детерминированных возмущений с мажорантами $\eta(t, \omega)$, у которых ограничены $I_{\eta}(\omega), \eta(\omega)$, доказательство устойчивости на этом заканчивается.

Случайные возмущения содержат дополнительный параметр $\omega \in \Omega$. Поэтому для семейства траекторий $\mathbf{x}_{g}(t, \omega)$ принадлежность окрестности $|\mathbf{x}|<\varepsilon$ можно обеспечить при тех возмущениях $\mathbf{g}(\mathbf{x}, t, \omega)$, для которых границы $\delta_{\eta}(\varepsilon, \omega) \geqslant \delta>0, \Delta_{\eta}(\varepsilon, \omega) \geqslant \Delta>0$ отделены от нуля равномерно по g. Гарантировать такую отделимость для возмущений со случайными мажорантами $\eta(t, \omega)$ не возможно. Но можно оценить множество неподходящих мажорант, используя конечность математического ожидания в условии (5).

Для возмущений $\mathbf{g}(\mathbf{x}, t, \omega)$, у которых мажоранты обладают свойствами $\exp \left(I_{\eta}(\omega)\right) \leqslant$ $R_{2} / \nu$, получаются оценки снизу:

$$
\delta_{\eta}(\varepsilon, \omega)>\frac{\varepsilon \nu}{R_{2}} \sqrt{\frac{m_{-}}{m_{+}}} \stackrel{\text { def }}{=} \delta(\varepsilon, \nu), \quad \Delta_{\eta}(\varepsilon, \omega)>\frac{\varepsilon \nu^{2}}{R_{1} R_{2}} \frac{m_{-}}{m_{1}} \sqrt{\frac{m_{-}}{m_{+}}} \stackrel{\text { def }}{=} \Delta(\varepsilon, \nu) .
$$

Значит, для возмущений, у которых мажоранты удовлетворяют неравенствам $\eta(\omega) \leqslant R_{1} / \nu$ и $\exp \left(I_{\eta}(\omega)\right) \leqslant R_{2} / \nu$, траектории $\mathbf{x}_{g}(t, \omega)$ с начальными точками $\left|\mathbf{x}_{0}\right|<\delta(\varepsilon, \nu)$ при $|\mu|<$ $\Delta(\varepsilon, \nu)$ не выходят из шара $|\mathbf{x}|<\varepsilon$.

Все оставшиеся возмущения со свойствами мажорант: $\eta(\omega)>R_{1} / \nu$ либо $\exp \left(I_{\eta}(\omega)\right)>$ $R_{2} / \nu$, составляют множество $\widehat{\mathscr{G}}$. Поскольку $\eta(\omega), \exp \left(I_{\eta}(\omega)\right)>0$, для этих случайных величин выводятся неравенства (см. [2; с. 32])

$$
\mathrm{P}\left(\eta(\omega)>\frac{R_{1}}{\nu}\right)<\frac{\mathrm{M}[\eta(\omega)]}{R_{1} / \nu}<\nu, \quad \mathrm{P}\left(\exp \left(I_{\eta}(\omega)\right)>\frac{R_{2}}{\nu}\right)<\frac{\mathrm{M}\left[\exp \left(I_{\eta}(\omega)\right)\right]}{R_{2} / \nu}<\nu .
$$

Траектории $\mathbf{x}=\mathbf{x}_{g}(t, \omega)$, для которых выбираются малыми начальное значение $\left|\mathbf{x}_{0}\right|<$ $\delta(\varepsilon, \nu)$ и параметр возмущения $|\mu|<\Delta(\varepsilon, \nu)$, но которые, тем не менее, не остаются в шаре $|\mathbf{x}|<\varepsilon$, обязаны быть решениями системы (2) с возмущениями из множества $\widehat{\mathscr{G}}$. Для них $\mathrm{P}\left(\sup _{t>t_{0}}\left|\mathbf{x}_{g}(t, \omega)\right|>\varepsilon\right)<\nu$. Поскольку числа $\varepsilon, \nu>0$ произвольные, теорема доказана.

ПримеР. Для уравнения $\dot{x}=x(x-1)$ равновесие $x=0$ устойчиво относительно возмущения в виде высоких случайных всплесков: $\mu g=G(\omega) / \mu$ на узком (по $t$ ) носителе длиной $\mu^{2}$. Здесь $\eta(\omega) \equiv 0, \mathrm{M}\left[I_{\eta}(\omega)\right]=\mathrm{M}[|G(\omega)|]<\infty$.

Автор благодарит проф. Насырова Ф. С. за обсуждение. 


\section{СПИСОК ЦИТИРОВАННОЙ ЛИТЕРАТУРЫ}

[1] Л. А. Калякин, УМН, 63:5 (2008), 3-72. [2] Р. З. Хасьминский, Устойчивость систем дифференииальных уравнений при случайных возмущениях их параметров, Наука, М., 1969. [3] М. М. Хапаев, Асимптотические методы и устойчивость в теории нелинейных колебаний, Высшая школа, М., 1988. [4] Р. Беллман, Теория устойчивости решений дифференииальных уравнений, ИЛ, М., 1954. [5] I. Vrcoč, Czechoslovak Math. J., 9(84) (1959), 71-129. [6] Н.Н. Красовский, Некоторые задачи теории устойчивости движения, Физматгиз, М., 1959.

Л. А. Калякин

Поступило

Институт математики с вычислительным центром

Уфимского научного центра РАН

E-mail: klenru@mail.ru 